The need for co-ordinating all grasshopper research has been repeatedly stressed by the Committee on Grasshopper Research, but the impression was that this is an aspiration rather than a demand for a practical measure. It would be natural to suggest that co-ordination of grasshopper research might be organised, or at least financed, jointly by the Govern. ments of the two North American countries, but somehow this appeared to be impracticable.

In spite of all handicaps, American entomologists are making great strides in the study of the grass. hopper problem. Unfortunately, their achievements are insufficiently known, since surprisingly little has been published on the subject, mainly because the routine work leaves no time for analysing and summarizing the results. It was even more surprising to hear that many important papers have been ready and waiting for publication for some years. Lack of funds as an explanation did not sound very con. vincing for a European visiting the United States. The Committee on Grasshopper Research has passed a very strong recommendation that the analysis of accumulated data and the publication of results should be speeded up; but it remains to be seen what the official reaction will be.

It is impossible here to discuss details which would be of interest only to specialists, who have a great deal to learn from the experience of American colleagues, particularly with regard to the technique and the organisation of grasshopper control. A much wider interest is attached to the general aspects of the North American grasshopper problem and its treatment. There is no doubt whatever that the problem is a man-made one, and it reached its great economic importance as a result of land development which was rapid, extensive and unscientific. A rapid expansion of unplanned agriculture in the North American continent has created exceptionally favourable conditions for various insect pests and for grasshoppers in particular. An analogous situation is rapidly developing in Australia, and it is only a matter of time before Africa will be faced with similar results of extensive agricultural development. There is in Africa a vast and varied grasshopper fauna, many members of which will undoubtedly develop into dangerous pests as soon as suitable soil conditions and abundant food are provided for them by the clearing of forests and by mechanized agriculture. At present, African development is threatened by locust plagues, which are a serious factor, but a periodical one; grasshopper plagues, although less spectacular than locust invasions, will be much more serious in their aggregate effects because they tend to be perennial, as the example of North America shows. Anti-locust policy in Africa is developing on the lines of prevention, and it became possible to formulate such policy only after some twenty years of international research. It will take a long time to study the possible effects of mechanized agriculture on native grasshoppers, and it is therefore not too early to consider commencing such studies. The alternative is to do nothing until the danger becomes imminent, by which time it may be too late for any measures of prevention, and perennial artificial control will be required. Again, the American example shows that such control is very expensive and only highly remunerative crops can stand the cost. Agricultural development of Africa is now being planned on a vast scale, and it may be wise not to forget possible handigaps.

\section{ATMOSPHERIC TURBULENCE}

ANY workers in atmospheric turbulence prob1 ably believe the subject to be more readily capable of formal and exact development than some other branches of dynamical meteorology, a belief deriving from a certain measure of success already attained. But that this success is rather slight is evident when one considers that there is still only slight understanding of turbulence in isothermal flow such as occurs in wind tunnels, pipes, etc., while the atmospheric problems are complicated by the existence of buoyancy forces, positive or negative, by the earth's rotation, and, over the oceans, by the form of the surface being dependent on the air motion itself. Again, there is a very considerable difference in scale between laboratory and atmospheric flow; but this difference may well be ultimately profitable to an improved understanding of turbulence on all scales-solar, terrestrial (atmospheric and oceanic), and laboratory.

G. I. Taylor effectively established the subject of atmospheric turbulence in his classical paper of 1915 . From observations of temperature on the Eiffel Tower and by kite over the Newfoundland Grand Banks, and from Dobson's observations of wind speed and direction in the first kilometre or so over Salisbury Plain, Taylor deduced that the turbulence of the lower atmosphere resulted in an effective coefficient of diffusion of heat or of momentum some $10^{4}-10^{6}$ times greater than the value for molecular diffusion, the ratio increasing with the wind speed and the lapse-rate of temperature. The equations of heat or momentum transport by which these results were obtained were solved by assuming the turbulent diffusivity constant through the layer concerned, whereas on any simplified picture of eddy motion a variation with height appeared probable; and this variation was involved in the next major advance in the subject early in the last decade. The advance was derived from Prandtl's application of mixing-length ideas growing out of earlier work by Taylor and Schmidt, the mixing-length being an analogue in eddy motion of the mean free path of molecular theory and connoting the mean length of path over which turbulent elements move between birth and extinction, that is, between aequiring and losing a velocity different from that of the mean motion at the levels in question. Prandtl assumed that near a boundary the mixing-length increased linearly with distance from the boundary, and by assuming further that the turbulent elements conserved their momentum during motion (that is, pressure field of no immediate consequence) he deduced that the mean velocity should increase with the logarithm of the distance from the boundary in the region of negligible variation of turbulent shearing stress. This result was in accord with observations in the laboratory for a very wide range of Reynolds number, and in the first $10 \mathrm{~m}$. or so of the atmosphere when in near-neutral static equilibrium. The mixinglength distance relation, more or less confirmed by this agreement of theory with observation, implied that turbulent diffusivity increased linearly with distance from the boundary and was given directly, for momentum transfer at least, from observations of the velocity profile.

Early in the 1920's, L. F. Richardson had examined the effect of static stability on the rate of growth or decay of turbulence. He showed that the rate of production of turbulent energy due to a velocity 
gradient would compensate the rate of decay due to the work done by eddies against (negative) buoyancy forces, provided the ratio of the square of the vertical gradient of mean velocity to the difference between the adiabatic and actual lapse-rates of mean temperature exceeded a certain critical magnitude. This criterion is only now receiving the attention and application which it merits.

It is to be noted that the above achievements are expressed in the main in terms of the bulk properties of the medium (mean speed and gradients of mean speed and of temperature, etc.), a desirable feature for a ready assessment of the diffusive properties of a given field of mean flow, but inadequate for an understanding of the mechanics of turbulence. Progress in the latter has been rather slight, though much experimental work has been done in the wind tunnel on the measurement of turbulent energy and of its spectrum, and on the correlation between various turbulent velocity components. This has been allied with a promising theoretical treatment of the statistical mechanics of isotropic turbulence, initiated by Taylor in 1935. A little knowledge has been gained of the statistics of atmospheric turbulence in the lower atmosphere, notably by Giblett, Scrase and Best in Great Britain, but there is very little information for the free atmosphere.

This was the background against which the Geophysical Discussion at the Royal Astronomical Society on "Atmospheric Turbulence" was held on October 24. Prof. O. G. Sutton opened the discussion by stressing the alternative approaches to the subject, the approach through the study of bulk properties on one hand and through the study of the internal structure of the fluid on the other, recapitulating the history of the subject and some of the more readily observed characteristics of atmospheric turbulence in illustration. A potentially profitable approach on the structural side was made by Taylor in 1922 in providing a relation between the diffusion of an entity and the correlation between the eddy velocities of a given element of fluid at increasing intervals of time. Sutton developed this treatment by an empirical formulation of the correlation coefficient (direct observations of the correlation coefficient would be very difficult and an a priori derivation presupposes a basic understanding of turbulence), and he deduced the rate of transfer of matter (evaporation) from a plane boundary in a turbulent flow, obtaining good agreement with observations for both matter and heat transfer in the laboratory for aerodynamically smooth flow, that is, flow in which the molecular viscosity is responsible for momentum transfer at the boundary itself. This treatment remains to be generalized for aerodynamically rough flow, that is, flow in which the geometrical roughness of the boundary is a controlling factor, and for an atmosphere statically stable or unstable. Sutton also pointed out some of the major meteorological problems of a larger scale requiring investigation and solution, such as the temperature and humidity distribution in the layer which is responsible for the propagation of short radio waves over the earth's surface (see Nature, 157, $860 ; 1946$ ), and the structure and distribution throughout the troposphere of gusts which on occasion may be a serious hazard for aircraft.

Prof. P. A. Sheppard spoke of his simultaneous measurements of surface drag and the accompanying velocity profile in the air. In the laboratory the mean velocity $u$ at distance $z$ from a boundary is related with the drag $\tau_{0}$ at the boundary by

$$
\begin{array}{r}
u=\frac{\left(\tau_{0} / \rho\right)^{1 / 2}}{k}\left[\log _{e}\left\{\frac{\left(\tau_{0} / \rho\right)^{1 / 2} z}{\nu}\right\}+1 \cdot 75\right] \\
\text {. . aerodynamically smooth flow, }
\end{array}
$$

or

$u=\frac{\left(\tau_{0} / \rho\right)^{1 / 2}}{k} \log _{e}\left(\frac{z}{z_{0}}\right)$

. . aerodynamically rough flow,

where $k$ (about 0.40 ) is the constant of proportionality between mixing length and distance referred to above, $\rho$ is the fluid density, $\nu$ the kinematic viscosity and $z_{0}$ a length characterizing the roughness of the boundary. For either type of flow the turbulent diffusivity $K\left(=\frac{\tau_{0} / \rho}{d u / d z}\right)$ is given by

$$
K=\left(\tau_{0} / \rho\right)^{1 / 2} k z,
$$

where $\tau_{0} / \rho$ may be obtained from observations of the velocity at two heights. Sheppard's observations were made to determine whether the same relation between profile and drag held in the lower atmosphere, or, alternatively stated, to determine the value of $k$ there. The result, for a moderately unstable atmosphere, was $k=0.45$, in very reason. able agreement with the laboratory value in view of the instability which would enhance $k$. There was also found to be a significant departure from linearity between mixing-length and height in the sense of larger mixing-lengths at the upper levels of observation-again reasonable in view of the instability arising from buoyancy. Correspondingly, one might expect a departure from linearity in the opposite sense in stable conditions. Such departures must be reflected in corresponding departures from the logarithmic profile of wind velocity. Further, if the mixing-length is the same for any entity diffusing vertically, the profile of that entity should show a variation with stability (Richardson's Number is likely to be the relevant parameter) similar to that for wind velocity. Evidence of such variation was shown in the profiles of wind speed, temperature and vapour pressure obtained by various workers in the first $15 \mathrm{~m}$. or so of the atmosphere; entities increased or decreased less rapidly than the logarithm of the height in unstable conditions, and more rapidly than this in stable conditions. Such profiles imply that the turbulent diffusivity increases.more rapidly than the height in unstable conditions and less rapidly than the height in stable conditions. Up to what level these relations apply is not yet known-the increases cannot continue indefinitely.

Mr. F. Pasquill spoke on turbulence in agricultural meteorology and dealt first with the loss of water from soil and crops by turbulent diffusion. If the diffusivity for matter is the same as for momentum, the former is immediately deducible from a velocity profile; and the rate of evaporation $E$ in a steady state (vertical flux of water vapour independent of height) is then obtainable from the vertical gradient of specific humidity $q(E=K / p \cdot d q / d z)$. Pasquill's results from observations of the velocity and humidity profiles in the first $2 \mathrm{~m}$. over grass gave quite encouraging agreement with the loss of water determined by soil sampling before and after the profiles had been determined, though the results of the soil sampling were a little ambiguous. Another agricultural application related to the dispersal of wind- 
borne spores, a problem first tackled by Schmidt many years ago. The spores are dispersed by turbulence as they travel down-wind and are simultaneously deposited on the ground due to fall under gravitya procedure analogous to the transport of silt in a river or estuary. Pasquill has calculated the rate of deposition at various distances down-wind of the source under given conditions, and obtains quite reasonable agreement with the rather meagre experimental data available.

Mr. K. L. Calder presented some very striking evidence of the applicability of Prandtl's expression for the eddy diffusivity in an isothermal boundary layer to the problem of the diffusion of gas and smoke emitted into the atmosphere near the ground along a line normal to the wind direction. The treatment takes account of the roughness of the underlying surface and gives excellent agreement with a large number of observed concentrations of gas and smoke.

Mr. E. L. Deacon showed a slide of velocity gradients, taken between $2 \mathrm{~m}$. and $4 \mathrm{~m}$. at a given site in various wind speeds and static stabilities, plotted against the Richardson Number for the same height-interval and times of observations. The high correlation shown gave strong support to the growing realization of the importance of Richardson's parameter in atmospheric turbulence.

The 'structural' approach to the study of atmospheric turbulence, either in terms of Prandtl's mixing-length or of Sutton's application of the Taylor correlation coefficient, has been essentially empirical. The mixing-length as used by Prandtl is indeed an idealized concept corresponding to no measurable property of the turbulence itself; it is rather a parameter with the dimension of length which appears from a suitable transformation of the turbulent flux equation. Sutton ascribed the difficulty of developing an a priori treatment of turbulence to the non-linear form of the Navier-Stokes equations and saw little hope of overcoming this difficulty, but Mr. G. K. Batchelor ended the dis. cussion on a more hopeful note. He instanced the appreciable success of the fundamental theory launched by Taylor in 1935 to deal with the correlations between eddy velocities at points across and along the direction of mean flow when the turbulence is isotropic, and with the decay of such turbulence in a free stream. The theory is still in vigorous development, and may be expected, sooner or later, to provide better understanding of atmospheric problems.

P. A. Sheppard

\section{NEWS and VIEWS}

\section{Students in British Universities}

IN NATURE of December 6, in the course of an article on "University Expansion in Great Britain", a statement is attributed to Lord Simon to the effect that the output of arts graduates has risen more rapidly than that of science graduates, due to relatively greater ease of expansion on the arts side. Against this remark should be set the figures for university 'populations' given in a written answer by Sir Stafford Cripps in the House of Commons (Hansard, House of Commons, December 1). The total number of full-time students at university institutions in Great Britain for the autumn term 1947 was 76,764 , compared with 50,246 for the academic year 1938-39. Analysing these totals by faculties, the figures for arts students were 34,200 and 22,512 respectively. The figures under pure science are 14,520 for 1947 and 7,767 for 1938-39 ; and an approximate doubling is also shown under technology $(10,143$ and 5,288$)$ and under agriculture (2,337 and 1,043). Thus, whatever may be the present position with regard to the employment of arts graduates, the actual numbers of students in the arts faculties and in the science and technological faculties now in British universities have increased by about the same numbers since 1938-39, namely, 11,688 and 12,902 respectively; but whereas this represents an increase in the arts faculties of 52 per cent, in the science and technology faculties the increase is 91.5 per cent.

Some interesting figures are also given regarding the numbers of students now at the various universities of Britain as compared with the figures for the year 1938-39. Taking the larger universities only, they are as follow, the number in parenthesis being that for the last pre-war year: Birmingham, 2,975 (1,433); Bristol, 1,902 (1,005); Cambridge, 6,943 (5,931); Durham, 3,773 (1,709); Leeds, 2,888 (1,757); Liverpool, 2,943 (2,055) ; London, 15,789 $(13,191)$; Manchester, 4,632 (2,462); Nottingham,
1,679 (582); Oxford, 7,500 (5,023) ; Reading, 990 (584) ; Sheffield, 1,670 (767) ; Wales, 4,654 (2,779); Aberdeen, 2,007 (1,211); Edinburgh, 4,904 (3,205); Glasgow, 7,073 (4,690); St. Andrews, 1,811 (928). The increases have thus been well spread over the whole country, though the biggest percentage inereases are shown by some of the smaller institutions.

\section{Annals of Botany: Prof. V. H. Blackman}

READERS of the Annals of Botany will have seen with an element of regret that since September 30 Prof. V. H. Blackman has ceased to be editor. Prof. W. H. Pearsall, who succeeds him, is preeminently well qualified to do so, and as former editor of the Journal of Ecology is well known to many botanical authors. It is, however, fitting to record the very great debt which the botanical world owes to Prof. Blackman who, for more than twentyfive years, has borne with equanimity, kindliness and wisdom the editorship of one of the most influential botanical journals in the world. Almost every working botanist in Great Britain, and many from overseas, have at some time in their lives come under his influence, and the blue pencil has always been administered so graciously that a permanent friend. ship with the author has generally resulted in spite of compulsory improvement to literary style. It is greatly to be hoped that Prof. Blackman's influence on botany as a whole, and on younger botanists in particular, will continue to be felt for many years in other ways after the very considerable burden of the editorship has been lifted from him.

\section{Sir Evelyn Shaw, K.C.V.O., and the Exhibition of 1851 Scholars}

An interesting informal gathering took place at the offices of the Royal Commission for the Exhibition of 1851 on December 5. A number of former ' 1851 ' scholars made a presentation to Sir Evelyn Shaw, 